\title{
Joint measurability and temporal steering
}

\author{
H. S. Karthik, ${ }^{1,6}$ J. Prabhu Tej, ${ }^{2,7}$ A. R. Usha Devi, ${ }^{2,3, *}$ and A. K. Rajagopal ${ }^{3,4,5,8}$ \\ ${ }^{1}$ Raman Research Institute, Bangalore 560 080, India \\ ${ }^{2}$ Department of Physics, Bangalore University, Bangalore 560 056, India \\ ${ }^{3}$ Inspire Institute Inc., Alexandria, Virginia 22303, USA \\ ${ }^{4}$ Harish-Chandra Research Institute, Chhatnag Road, Jhunsi, Allahabad 211 019, India \\ ${ }^{5}$ Institute of Mathematical Sciences, C.I.T. Campus, Taramani, Chennai 600113, India \\ ${ }^{6}$ e-mail: karthik@rri.res.in \\ 7e-mail: j.prabhutej@gmail.com \\ ${ }^{8}$ e-mail: attipat.rajagopal@gmail.com \\ *Corresponding author: arutth@rediffmail.com
}

Received 3 December 2014; revised 4 March 2015; accepted 5 March 2015; posted 12 March 2015 (Doc. ID 228916); published 26 March 2015

\begin{abstract}
Quintino et al. [Phys. Rev. Lett. 113, 160402 (2014)] and Uola et al. [Phys. Rev. Lett. 113, 160403 (2014)] have recently established an intrinsic relation between nonjoint measurability and Einstein-Podolsky-Rosen steering. They showed that a set of measurements are incompatible (i.e., not jointly measurable) if and only if they can be used for the demonstration of steering. In this paper, we prove the temporal analog of this result, viz., a set of measurements are incompatible if and only if they exhibit temporal steering in a single quantum system. (C)2015 Optical Society of America
\end{abstract}

OCIS codes: (270.5585) Quantum information and processing; (000.1600) Classical and quantum physics; (000.2658) Fundamental tests.

\section{INTRODUCTION}

The purpose of measurements is to discern the properties of a system under investigation. In the classical scenario, all the physical observables are jointly measurable (or compatible). In contrast, in the quantum world, noncommuting observables are declared to be incompatible, because it is not possible to assign well-defined values to these observables jointly. The notion of compatibility of measurements is captured entirely by commutativity of the observables if one restricts them to only projective valued (PV) measurements. However, connecting compatibility of measurements with commutativity turns out to be limited in an extended framework, where the conventional idea of sharp PV measurements of selfadjoint observables gets broadened to include generalized measurements of positive operator valued observables [1-11]. Active research efforts are dedicated $[\underline{1}, \underline{4}-18]$ to investigating clear operational criteria of joint measurability within the generalized framework of positive operator valued measures (POVMs) and to identify the significance of incompatible measurements in revealing puzzling quantum features like Bell nonlocality [19], contextuality [20], and steering [21,22]. Particularly, Wolf et al. [8] have proved that the violation of the Clauser-Horne-Shimony-Holt [23] inequality in an entangled state can be witnessed if and only if incompatible measurements of any pairs of POVMs with binary outcomes are employed. It has been realized that a generalized noncontextuality inequality can be violated by a quantum state in two-dimensional [24] Hilbert space by employing a set of three dichotomic POVMs that have pairwise joint measurability - but not triplewise joint measurability $[11,14]$. In yet another recent development, Quintino et al. [16] and Uola et al. [17] have established that a set of nonjointly measurable POVMs is the one that is useful for the task of nonlocal steering.

The concept of nonlocal Einstein-Podolsky-Rosen (EPR) steering was originally initiated by Schrödinger [21] -as the ability to remotely prepare the state of a subsystem of an entangled state by performing local measurements on another subsystem. An experimentally testable steering criterion was developed by Reid [25] for continuous variable systems (Reid proposed an uncertainty relation involving conditional variances of position and momentum violation that demonstrates EPR nonlocality in an entangled state; this is the first steering inequality, though it was not explicitly stated in Ref. [25]). Wiseman et al. [26] formalized the task of steering via falsification of local hidden state (LHS) models and showed that steering constitutes a different class of nonlocality that lies between entanglement and Bell nonlocality. 
The quantum steering task is usually described in terms of an example: Alice supposedly prepares a composite quantum state and sends a subsystem to Bob. She tries to convince Bob that they share an entangled state, which would allow her (with the help of local measurements on her part of the system) to remotely affect (steer) Bob's quantum state. In order to verify if Alice's claim is true, Bob asks Alice to perform a local measurement of an observable $X_{k}$ on her part of the state and announce her result $x_{k}$. From local quantum state tomography (via measurements at his end), Bob determines his set of states $\left\{\rho_{x_{k} \mid k} / \operatorname{Tr}\left[\rho_{x_{k} \mid k}\right]\right\}$. If Bob's assemblage [16], i.e., the set of unnormalized states $\left\{\rho_{x_{k} \mid k}\right\}$ (realized in each experimental run for different choices of Alice's observables $X_{k}$ with statistical outcomes $x_{k}$ ), does not admit a LHS decomposition of the form [27]

$$
\rho_{x_{k} \mid k}=\sum_{\lambda} g(\lambda) p\left(x_{k} \mid k, \lambda\right) \rho_{\lambda}
$$

(where $0 \leq g(\lambda) \leq 1 ; \sum_{\lambda} g(\lambda)=1$ and $0 \leq p\left(x_{k} \mid k, \lambda\right) \leq 1$; $\left.\sum_{x_{k}} p\left(x_{k} \mid k, \lambda\right)=1\right)$, then Bob can convince himself that Alice is not cheating him and they indeed share an entangled state. On the other hand, if Bob's assemblage $\left\{\rho_{x_{k} \mid k}\right\}$ admits a LHS structure [Eq. (1)], he would be unable to witness violation of any of the steering inequalities [22], and would therefore conclude that Alice is fooling him.

In order to demonstrate steerability, (i) Alice and Bob should necessarily share an entangled state (though the converse is not true [26]), and (ii) Alice's measurements must be comprised of incompatible POVMs $[16,17]$. Thus, in addition to entanglement being a resource for the steering task, incompatibility of measurements also plays a key role.

Apart from the developments toward probing nonlocality, in 1985 Leggett and Garg [28] proposed an inequality to test the concept of macrorealism in a single quantum system in terms of the correlations in the statistical outcomes of a dynamical observable at different times [29-33]. Macrorealism rests on the following assumptions: (i) physical properties of a macroscopic object exist independent of the act of observation (postulate on the existence of reality for all physical observables in the macroscopic world), and (ii) measurements are noninvasive, i.e., the measurements of an observable at different instants of time do not influence its subsequent dynamical evolution. It has been experimentally demonstrated in a wide range of quantum systems [34-39] that temporal correlations in the outcomes of an observable measured sequentially on a quantum system at different times do not fall under the tenet of macrorealism, i.e., they violate the Leggett-Garg inequality (also known as the temporal Bell inequality $[\underline{40}, \underline{41}])$. Very recently, the temporal analog of steering in a single quantum system has been proposed $[42,43]$. In this paper we show that the connection between spatial steerability and incompatible measurements of Refs. [16] and [17] can be extended to its temporal counterpart in a single quantum system also, viz., nonjoint measurability is necessary and sufficient for temporal steerability.

We organize the contents of the paper as follows. We begin by giving a concise description of joint measurability of POVMs in Section $\underline{2}$. Section $\underline{3}$ is devoted to discussing the temporal analog of EPR steering in a single quantum system and to establish that a set of incompatible POVMs is necessary and sufficient for temporal steering. An example to bring forth the intrinsic connection between temporal steering and incompatibility of measurements in a single qubit system is discussed in the Subsection 3.A. Concluding remarks are given in Section $\underline{4}$.

\section{JOINT MEASURABILITY OF POVMS}

Mathematically, a POVM is a collection $\mathbb{E}=\{E(x)\}$ consisting of positive self-adjoint operators $E(x) \geq 0$ as its elementswhich sum up to give the identity operator $\mathbb{1}$, i.e., $\sum_{x} E(x)=\mathbb{1}$. A measurement of $\mathbb{E}$ in a quantum state $\rho$ results in the outcomes $x$ with probability of occurrence $p(x \mid E)=$ $\operatorname{Tr}[\rho E(x)]$. It may be noted that the POVM $\{E(x)\}$ encompasses the conventional $\mathrm{PV}$ measurements as a special case [when the elements $E(x)$ of the POVM constitute a complete and orthogonal set $\{\Pi(x)\}$ of projectors].

In the restricted measurement scenario, where only PV measurements are considered, it is well established that commuting physical observables are jointly measurable. But in the extended framework of generalized measurements, the notion of joint measurability is nontrivial and has received increasing attention [1-11]. A more refined notion of compatible (jointly measurable) POVMs is defined as follows. A set of POVMs $\mathbb{E}_{k}=$ $\left\{E_{k}\left(x_{k}\right)\right\}$ is said to be compatible if the probabilities $p\left(x_{k} \mid k\right)=$ $\operatorname{Tr}\left[\rho E_{k}\left(x_{k}\right)\right]$ of their outcomes in any arbitrary quantum state $\rho$ can be discerned by measuring a global POVM $\mathbb{G}=$ $\left\{G(\lambda) ; G(\lambda) \geq 0, \sum_{\lambda} G(\lambda)=\mathbb{1}\right\}$-where the measurement outcomes $\lambda=\left\{x_{1}, x_{2}, \ldots\right\}$ of $G(\lambda)$ occur with probabilities $g(\lambda)=\operatorname{Tr}[\rho G(\lambda)]$-by classical postprocessing of the data $[\underline{7,}, \underline{17}]$

$$
p\left(x_{k} \mid k\right)=\sum_{\lambda} g(\lambda) p\left(x_{k} \mid k, \lambda\right)
$$

where $0 \leq p\left(x_{k} \mid k, \lambda\right) \leq 1$ are some arbitrary positive numbers satisfying $\sum_{x_{k}} p\left(x_{k} \mid k, \lambda\right)=1$.

More precisely, associated with a set of jointly measurable POVMs $\left\{E_{k}\left(x_{k}\right)\right\}$, there exists a grand POVM $\mathbb{G}=\{G(\lambda)\}$ such that

$$
E_{k}\left(x_{k}\right)=\sum_{\lambda} p\left(x_{k} \mid k, \lambda\right) G(\lambda) \quad \forall k .
$$

In other words, it suffices to measure the grand POVM $\mathbb{G}$ to discern the measurement results of compatible POVMs $\mathbb{E}_{k}$.

An important aspect to be highlighted here is that the generalized POVMs are jointly measurable even if they do not commute with each other.

Consider a triad of qubit observables $X=|0\rangle\langle 1|+| 1\rangle\langle 0|$, $Y=-i|0\rangle\langle 1|+i| 1\rangle\langle 0|$, and $Z=|0\rangle\langle 0|-| 1\rangle\langle 1|$, measured by employing the POVMs $\mathbb{E}_{X}, \mathbb{E}_{Y}$, and $\mathbb{E}_{Z}$, defined in terms of their elements:

$$
\begin{aligned}
& E_{X}(x)=\frac{1}{2}(\mathbb{1}+\eta x X), \\
& E_{Y}(y)=\frac{1}{2}(\mathbb{1}+\eta y Y), \\
& E_{Z}(z)=\frac{1}{2}(\mathbb{1}+\eta z Z) .
\end{aligned}
$$


The measurements result in binary outcomes $x, y, z= \pm 1$, and they correspond to fuzzy measurements of the observables $X, Y, Z$, characterized by the unsharpness parameter $0 \leq \eta \leq 1$. It has been identified that the qubit POVMs $\left\{E_{X}(x)\right\},\left\{E_{Y}(y)\right\}$, and $\left\{E_{Z}(z)\right\}$ are pairwise jointly measurable if and only if $\eta \leq 1 / \sqrt{2}$, and the condition $\eta \leq 1 / \sqrt{3}$ is necessary and sufficient for their triplewise joint measurability $[6,11,14]$. It may also be noted that when $\eta=1$, the POVMs $\left\{E_{X}(x)\right\},\left\{E_{Y}(y)\right\},\left\{E_{Z}(z)\right\}$ reduce to their corresponding sharp PV versions $\left\{\Pi_{X}(x)\right\},\left\{\Pi_{Y}(y)\right\},\left\{\Pi_{Z}(z)\right\}$.

\section{TEMPORAL STEERING AND INCOMPATIBLE MEASUREMENTS}

We consider a system prepared in a quantum state $\rho=\rho(0)$ that evolves under the Hamiltonian evolution $U(t)=e^{-i H t / \hbar}$, dynamically transforming the state (in the Schrödinger picture) as $\rho \rightarrow \rho(t)=U(t) \rho U^{\dagger}(t)$ at time $t$. The physical observables undergo dynamical evolution (in the Heisenberg picture) as $X(0) \rightarrow X(t)=U^{\dagger}(t) X(0) U(t)$. The observable $X$ at different time instants $t_{k}$ (which we denote by $X_{k}$ ) do not commute in general. Hence, $\left\{X_{k}\right\}$ values are not jointly measurable within the restricted framework of PV measurements. Contrast this situation with the classical scenario, where measurement of an observable at a given instant in time does not disturb its subsequent evolution. In other words, one can measure an observable at different instants in time jointly in the classical scenario. In the quantum case, measurements of noncommuting observables, in general, form an incompatible set of measurements.

To illustrate the temporal analog of steering, we consider a game involving two players, Alice and Bob. Alice prepares a state $\rho$ (which is not disclosed to Bob). Bob asks Alice to measure the observable $X$ at different instants of time $t_{k}$ using incompatible POVMs. Alice claims that she has measured $X_{k}$ and obtained an outcome $x_{k}$ with probability $p\left(x_{k} \mid k\right)$. She gives the postmeasured states $\frac{\rho_{x_{k} \mid k}}{\operatorname{Tr}\left[\rho_{x_{k} \mid k}\right]}$ to Bob. Bob's task is to verify whether Alice has given him a genuine set of postmeasured assemblage $\left\{\rho_{x_{k} \mid k}\right\}$, where the unnormalized states

$$
\rho_{x_{k} \mid k}=\sqrt{E_{k}\left(x_{k}\right)} \rho \sqrt{E_{k}\left(x_{k}\right)}
$$

have resulted via measurements of incompatible POVMs $\left\{E_{k}\left(x_{k}\right)\right\}$ of the observables $X_{k}$, or whether Alice is cheating him by merely stating that she has performed the measurements.

Bob can only trust his measurements on the states handed over to him by Alice, with a prelabel $\left\{x_{k}, p\left(x_{k} \mid k\right)\right\}$. In order to accomplish the protocol, Bob may choose to measure the observables $X_{l}$ at a later time, $l \geq k$, on the assemblage $\rho_{x_{k} \mid k}$ and record the conditional probabilities $\mathcal{P}\left(x_{l} \mid x_{k}\right)$ of his outcomes $x_{l}$ (given that Alice had obtained an outcome $x_{k}$ in her measurement of the observable $X_{k}$ ); he then explores whether the temporal correlations of the observables $X_{k}, X_{l}$ violate any steering inequality [44]. If the temporal steering inequality is violated, then Bob concludes that Alice has indeed performed incompatible measurements of the observables $X_{k}$. We refer to this scenario as temporal steering [45].

More generally, Bob could determine the assemblage $\left\{\rho_{x_{k} \mid k}\right\}$ given to him through quantum state tomography; if the assemblage $\rho_{x_{k} \mid k}$ is of the hidden state (HS) form, $\rho_{x_{k} \mid k}=\sum_{\lambda} g(\lambda) p\left(x_{k} \mid k, \lambda\right) \rho_{\lambda}$ (which is identical to the LHS form [Eq. (1)]), where $0 \leq p\left(x_{k} \mid k, \lambda\right) \leq 1, \sum_{x_{k}} p\left(x_{k} \mid k, \lambda\right)=1$, then Bob convinces himself that the assemblage $\left\{\rho_{x_{k} \mid k}\right\}$ is not temporally steered. This is because the actual scenario may be the following. Alice could have drawn some random states $\rho_{\lambda}$ with probability $g(\lambda)$ (from a statistical mixture $\rho=\sum_{\lambda} g(\lambda) \rho_{\lambda}$ ) but announce that an outcome $x_{k}$ has occurred in the measurement of the observable $X$ at time $t_{k}$, with a probability of occurrence $p\left(x_{k} \mid k, \lambda\right) \quad$ [Alice could have theoretically calculated the probabilities $p\left(x_{k} \mid k, \lambda\right)$ for the hypothetical outcomes $x_{k}$ of measurement]. If Alice has indeed performed incompatible measurements, as she claims, Bob's assemblage $\left\{\rho_{x_{k} \mid k}\right\}$ deviates from the HS form. Bob can then convince himself that Alice has indeed given him a set of states that reveals temporal steering, and it has resulted from the measurements of the observable $X$ at different instants in time using incompatible POVMs.

We now proceed to show that measurements of $\left\{X_{k}\right\}$ using a compatible set $\left\{\mathbb{E}_{k}\right\}$ of POVMs do not lead to temporal steering.

Let us suppose that Alice performs the measurement of a global POVM $\mathbb{G}=\{G(\lambda)\}$. After her measurement, the postmeasured states are given by

$$
\rho_{\lambda}=\sqrt{G(\lambda)} \rho \sqrt{G(\lambda)} / g(\lambda),
$$

where $g(\lambda)=\operatorname{Tr}[\rho G(\lambda)]$ is the probability of outcome $\lambda$. Alice would then classically postprocess the measurement data of the global POVM $\mathbb{G}=\{G(\lambda)\}$ to obtain the probabilities of outcomes $p\left(x_{k} \mid k\right)$ of measurement of any compatible POVMs $\mathbb{E}_{k}$ to have resulted in an outcome $x_{k}$ as

$$
\begin{aligned}
p\left(x_{k} \mid k\right) & =\operatorname{Tr}\left[\rho E_{k}\left(x_{k}\right)\right] \\
& =\sum_{\lambda} p\left(x_{k} \mid k, \lambda\right) \operatorname{Tr}[\rho G(\lambda)] \\
& =\sum_{\lambda} p\left(x_{k} \mid k, \lambda\right) g(\lambda) .
\end{aligned}
$$

More specifically, Alice could discern the results of measurements of compatible POVMs $\mathbb{E}_{k}=\left\{E_{k}\left(x_{k}\right)\right\}$ via measurement of a global POVM $\mathbb{G}=\{G(\lambda)\}$ and then use of the decomposition $E_{k}\left(x_{k}\right)=\sum_{\lambda} p\left(x_{k} \mid k, \lambda\right) G(\lambda)$.

After Alice announces her measurement results $\left\{x_{k}, p\left(x_{k} \mid k\right)\right\}$ of $E_{k}\left(x_{k}\right)$ and hands over the postmeasured set of states, Bob detects that his assemblage $\left\{\rho\left(x_{k} \mid k\right)\right\}$ is of the HS form $\rho\left(x_{k} \mid k\right)=\sum_{\lambda} g(\lambda) p\left(x_{k} \mid k, \lambda\right) \rho_{\lambda}$. Thus, Bob concludes that there is no temporal steering.

Conversely, we prove that nonjointly measurable (incompatible) POVMs are sufficient to demonstrate temporal steering. To demonstrate, we consider a completely random state $\rho=1 / d$ and a set of POVMs $\left\{\mathbb{E}_{k}\right\}$ for the measurements of the observables $\left\{X_{k}\right\}$. The postmeasured assemblage $\left\{\rho_{x_{k} \mid k}\right\}$ is characterized by its elements,

$$
\rho_{x_{k} \mid k}=\sqrt{E_{k}\left(x_{k}\right)} \rho \sqrt{E_{k}\left(x_{k}\right)}=\frac{1}{d} E_{k}\left(x_{k}\right) .
$$

One can thus express the elements $E_{k}\left(x_{k}\right)$ of the POVM in terms of the assemblage $\left\{\rho_{x_{k} \mid k}\right\}$ as [늘

$$
E_{k}\left(x_{k}\right)=d \rho_{x_{k} \mid k} \text {. }
$$

If there is no temporal steering, then the assemblage $\left\{\rho_{x_{k} \mid k}\right\}$ is described by a HS form [Eq. (1)], and hence one obtains 


$$
E_{k}\left(x_{k}\right)=d \sum_{\lambda} g(\lambda) p\left(x_{k} \mid k, \lambda\right) \rho_{\lambda}=\sum_{\lambda} p\left(x_{k} \mid k, \lambda\right) G(\lambda),
$$

where $G(\lambda)=d g(\lambda) \rho_{\lambda}$. It is easy to see that Eq. (10) is essentially the joint measurability condition [see Eq. (3)] for the definition of compatible POVMs $\left\{E_{k}(x)\right\}$. We thus obtain the result: a set of POVMs is said to be nonjointly measurable if and only if it is useful for demonstrating temporal steering.

Our result highlights that one does not require a steerable entangled state to determine whether a given set of measurements is compatible or not; it suffices to detect temporal nonsteerability in a single quantum system itself to accomplish this task.

\section{A. Joint Measurability and Temporal Steering in a Single Qubit System}

For the purpose of illustrating the intrinsic connection between temporal steering and nonjoint measurability of the POVMs in a single qubit system, suppose that Alice prepares a single qubit system in a maximally disordered state $\rho=\frac{1}{2}$. Bob asks Alice to subject the system to a Hamiltonian evolution $U(t)=e^{-i H t / \hbar}$ where the Hamiltonian $H=\hbar \omega Z=\hbar \omega(|0\rangle\langle 0|-| 1\rangle\langle 1|)$ and measure the observables $X_{k}=U^{\dagger}\left(t_{k}\right) X U\left(t_{k}\right)=$ $X \cos \left(\omega t_{k}\right)+Y \sin \left(\omega t_{k}\right) ; \quad X=|0\rangle\langle 1|+| 1\rangle\langle 0|, \quad Y=$ $-i(|0\rangle\langle 1|-| 1\rangle\langle 0|)$ at two different time intervals (i) $t_{1}=0$ and (ii) $t_{2}=\pi /(2 \omega)$ using incompatible POVMs. Alice employs binary outcome POVMs $\left\{E_{X}(x)=\frac{1}{2}(\mathbb{1}+\eta x X), x=\right.$ $\pm 1\}$ and $\left\{E_{Y}(y)=\frac{1}{2}(\mathbb{1}+\eta y Y), y= \pm 1\right\}$ to measure the observables $X_{1}=X$ and $X_{2}=Y$, respectively. After her measurements on several identically prepared copies of the initial state, Alice hands over four different assemblages $\left\{\rho_{x= \pm 1 \mid 1}\right\}$, $\left\{\rho_{y= \pm 1 \mid 2}\right\}$-labeled by the outcomes of measurements $x= \pm 1, y= \pm 1$ - and the corresponding probablilities of occurrence $p(x= \pm 1 \mid 1), p(y= \pm 1 \mid 2)$ to Bob. Bob then chooses to perform PV measurement $\left\{\Pi_{X}\left(x^{\prime}\right)=\frac{1}{2}\left(\mathbb{1}+x^{\prime} X\right) ; x^{\prime}= \pm 1\right\}$ at time $t_{3}=2 \pi / \omega$ on the assemblage $\left\{\rho_{x= \pm 1 \mid 1}\right\}$ (note that at $t_{3}=2 \pi / \omega$, the observable $X_{3}=X$ ); he obtains the conditional probabilities for his measurement outcomes $x^{\prime}= \pm 1$ :

$$
\begin{aligned}
\mathcal{P}\left(x^{\prime} \mid x\right) & =\operatorname{Tr}\left[\rho_{x \mid 1} \Pi_{X}\left(x^{\prime}\right)\right] / p(x) \\
& =\operatorname{Tr}\left[E_{X}(x) \Pi_{X}\left(x^{\prime}\right)\right] \\
& =\frac{1}{2}\left(1+\eta x x^{\prime}\right) .
\end{aligned}
$$

Further, Bob carries out PV measurements $\left\{\Pi_{Y}\left(y^{\prime}\right)=\frac{1}{2}(\mathbb{1}+\right.$ $\left.\left.y^{\prime} Y\right) ; y^{\prime}= \pm 1\right\}$ at time $t_{4}=5 \pi /(2 \omega)$ on the assemblage $\left\{\rho_{y= \pm 1 \mid 1}\right\}$ (at $t_{4}=5 \pi /(2 \omega)$, the observable $X_{4}=Y$ ) and registers the conditional probabilities for his measurement outcomes $y^{\prime}= \pm 1$ :

$$
\begin{aligned}
\mathcal{P}\left(y^{\prime} \mid y\right) & =\operatorname{Tr}\left[\rho_{y \mid 2} \Pi_{Y}\left(y^{\prime}\right)\right] / p(y), \\
& =\operatorname{Tr}\left[E_{Y}(y) \Pi_{Y}\left(y^{\prime}\right)\right] \\
& =\frac{1}{2}\left(1+\eta y y^{\prime}\right) .
\end{aligned}
$$

(In the second lines of Eqs. (11) and (12), we have substituted the probabilities of Alice's outcomes $p(x)=$ $\operatorname{Tr}\left[\rho E_{X}(x)\right]=1 / 2, p(y)=\operatorname{Tr}\left[\rho E_{Y}(y)\right]=1 / 2$ in the qubit state $\rho=\frac{1}{2}$.)
As the expectation value of the qubit observable $(X+Y) /$ $\sqrt{2}$ in any arbitrary qubit state is constrained to be less than 1 (the maximum eigenvalue of the observable), evidently the conditional expectation value of the observable $(X+Y) / \sqrt{2}$ (evaluated from Bob's measurement outcomes $x^{\prime}, y^{\prime}$ of $X$, $Y$-which are conditioned by Alice's POVM outcomes $x, y$ of the same observables) also is restricted, i.e.,

$$
\begin{aligned}
\left\langle\frac{(X+Y)}{\sqrt{2}}\right\rangle_{x, y} & =\frac{1}{\sqrt{2}}\left(\langle X\rangle_{x}+\langle Y\rangle_{y}\right) \\
& =\frac{1}{\sqrt{2}}\left[\sum_{x^{\prime}} \mathcal{P}\left(x^{\prime} \mid x\right) x^{\prime}+\sum_{y^{\prime}} \mathcal{P}\left(y^{\prime} \mid y\right) y^{\prime}\right] \leq 1 .
\end{aligned}
$$

If the assemblages $\left\{\rho_{x= \pm 1 \mid 1}\right\},\left\{\rho_{y= \pm 1 \mid 1}\right\}$, obtained after Alice performs her measurements, constitute a HS structure, i.e., $\quad \rho_{x= \pm 1 \mid 1}=\sum_{\lambda} g(\lambda) p(x \mid 1 ; \lambda) \rho_{\lambda} \quad$ and $\quad \rho_{y= \pm 1 \mid 1}=$ $\sum_{\lambda} g(\lambda) p(y \mid 1 ; \lambda) \rho_{\lambda}$, then Bob's measurements lead to a linear temporal steering inequality (obtained following the arguments outlined by Cavalcanti et al. [22] for the derivation of linear EPR steering criteria for two spatially separated qubits):

$$
\left|\sum_{x= \pm 1} p(x) x\langle X\rangle_{x}+\sum_{y= \pm 1} p(y) y\langle Y\rangle_{y}\right| \leq \sqrt{2} .
$$

Substituting the conditional probabilities in Eqs. (11) and (12) to evaluate the expectation values $\langle X\rangle_{x},\langle Y\rangle_{y}$ and simplifying, the linear temporal inequality [Eq. (14)] results in the constraint

$$
\eta \leq \frac{1}{\sqrt{2}}
$$

on the unsharpness parameter-which is exactly the condition for joint measurability $[\underline{6}, 11,47]$ of the qubit observables $X, Y$ using the POVMs $\left\{E_{X}(x)=\frac{1}{2}(\mathbb{1}+\eta x X) ; x= \pm 1\right\}$ and $\left\{E_{Y}(y)=\frac{1}{2}(\mathbb{1}+\eta y Y) ; y= \pm 1\right\}$. The temporal steering inequality [Eq. (14)] is violated for $\frac{1}{\sqrt{2}}<\eta \leq 1$, i.e., when Alice's POVMs are incompatible.

\section{CONCLUSIONS}

We have illustrated temporal steering phenomena in a single quantum system by developing the notion of a HS structurewhich is analogous to the LHS model for spatially separated systems. Falsification of the HS model implies temporal steerability. Extending the arguments of recent papers $[16,17]$, we have established a relation between the incompatibility of quantum measurements and temporal steering phenomena. Our results highlight that a set of measurements are incompatible if and only if they can be used to demonstrate temporal steering in any quantum state. The connection between measurement incompatibility and temporal steering opens up new avenues for exploring temporal steering inequalities to infer about (non) joint measurability. Further, following similar lines of investigation on nonlocal steering versus Bell nonlocality [26] of spatially separated states, it would be of interest to investigate whether Leggett-Garg inequalities and temporal steering inequalities carry identical inferences about measurement 
invasiveness [48], or whether they bring forth its different manifestations. We leave open these aspects for future investigations.

One of us (J. P.) acknowledges support from UGC-BSR, Government of India.

\section{REFERENCES AND NOTES}

1. P. Busch, "Unsharp reality and joint measurements for spin observables," Phys. Rev. D 33, 2253-2261 (1986).

2. P. Busch, P. Lahti, and P. Mittelstaedt, The Quantum Theory of Measurement (Springer, 1991).

3. P. Lahti, "Coexistence and joint measurability in quantum mechanics," Int. J. Theor. Phys. 42, 893-906 (2003).

4. E. Andersson, S. M. Barnett, and A. Aspect, "Joint measurements of spin, operational locality, and uncertainty," Phys. Rev. D 72, 042104 (2005).

5. W. Son, E. Andersson, S. M. Barnett, and M. S. Kim, "Joint measurements and Bell inequalities," Phys. Rev. A 72, 052116 (2005).

6. T. Heinosaari, D. Reitzner, and P. Stano, "Notes on joint measurability of quantum observables," Found. Phys. 38, 1133-1147 (2008).

7. S. T. Ali, C. Carmeli, T. Heinosaari, and A. Toigo, "Commutative POVMs and fuzzy observables," Found. Phys. 39, 593-612 (2009).

8. M. M. Wolf, D. Perez-Garcia, and C. Fernandez, "Measurements incompatible in quantum theory cannot be measured jointly in any other no-signaling theory," Phys. Rev. Lett. 103, 230402 (2009).

9. S. Yu, N. Liu, L. Li, and C. H. Oh, "Joint measurement of two unsharp observables of a qubit," Phys. Rev. A 81, 062116 (2010).

10. T. Heinosaari and M. M. Wolf, "Nondisturbing quantum measurements," J. Math. Phys. 51, 092201 (2010).

11. Y. C. Liang, R. W. Spekkens, and H. M. Wiseman, "Specker's parable of the overprotective seer: a road to contextuality, nonlocality and complementarity," Phys. Rep. 506, 1-39 (2011).

12. D. Reeb, D. Reitzner, and M. M. Wolf, "Coexistence does not imply joint measurability," J. Phys. A 46, 462002 (2013).

13. M. Banik, M. D. R. Gazi, S. Ghosh, and G. Kar, "Degree of complementarity determines the nonlocality in quantum mechanics," Phys. Rev. A 87, 052125 (2013)

14. R. Kunjwal and S. Ghosh, "Minimal state-dependent proof of measurement contextuality for a qubit," Phys. Rev. A 89, 042118 (2014).

15. R. Kunjwal, C. Heunen, and T. Fritz, "Quantum realization of arbitrary joint measurability structures," Phys. Rev. A 89, 052126 (2014).

16. M. T. Quintino, T. Vértesi, and N. Brunner, "Joint measurability, Einstein-Podolsky-Rosen steering, and Bell nonlocality," Phys. Rev. Lett. 113, 160402 (2014).

17. R. Uola, T. Moroder, and O. Gühne, "Joint measurability of generalized measurements implies classicality,” Phys. Rev. Lett. 113, 160403 (2014).

18. H. S. Karthik, A. R. Usha Devi, and A. K. Rajagopal, "Joint measurability, steering, and entropic uncertainty," Phys. Rev. A 91, 012115 (2015).

19. J. S. Bell, "On the Einstein Podolsky Rosen paradox," Physics 1, 195-200 (1964).

20. S. Kochen and E. P. Specker, "The problem of hidden variables in quantum mechanics," J. Math. Mech. 17, 59-87 (1967).

21. E. Schrödinger, "Discussion of probability relations between separated systems," Proc. Camb. Philos. Soc. 31, 555-563 (1935).

22. For a comprehensive historical outline, and for a formalization of the concept of quantum steering in the modern terminology, see E. G. Cavalcanti, S. J. Jones, H. M. Wiseman, and M. D. Reid, "Experimental criteria for steering and the Einstein-PodolskyRosen paradox," Phys. Rev. A 80, 032112 (2009).

23. J. F. Clauser, M. A. Horne, A. Shimony, and R. A. Holt, "Proposed experiment to test local hidden-variable theories," Phys. Rev. Lett. 23, 880-884 (1969)

24. Conventionally, the notion of quantum contextuality [20] is demonstrated by using projective measurements in Hilbert spaces of dimension $d \geq 3$.
25. M. D. Reid, "Demonstration of the Einstein-Podolsky-Rosen paradox using nondegenerate parametric amplification," Phys. Rev. A 40, 913-923 (1989).

26. H. M. Wiseman, S. J. Jones, and A. C. Doherty, "Steering, entanglement, nonlocality, and the Einstein-Podolsky-Rosen paradox," Phys. Rev. Lett. 98, 140402 (2007).

27. It could be that Alice randomly draws states $\rho_{\lambda}$ with probability $g(\lambda)$ from a chosen ensemble and sends it to Bob. And when Bob asks her to perform measurement of the observable $X_{k}$, she could communicate that a fake outcome $x_{k}$ occurred with the probability $p\left(x_{k} \mid k\right)=\sum_{\lambda} g(\lambda) p\left(x_{k} \mid k, \lambda\right)$ (based on her knowledge of a preexisting LHS model). In this scenario, Bob could detect that steering inequalities [22] are not violated-which in turn reveals that the LHS model [Eq. (1)] indeed holds.

28. A. J. Leggett and $\bar{A}$. Garg, "Quantum mechanics versus macroscopic realism: is the flux there when nobody looks?" Phys. Rev. Lett. 54 857-860 (1985).

29. Interestingly, correlations in the measurement outcomes of timeseparated observables in a single system mimic the nonlocal correlations in a spatially separated entangled system [see Č. Brukner, S. Taylor, S. Cheung, and V. Vedral, "Quantum entanglement in time," arXiv:quant-ph/0402127 (2004) and the following four references].

30. V. Vedral, "Using temporal entanglement to perform thermodynamical work," arXiv:1204.5559 (2012).

31. J. Kofler and Č. Brukner, "Condition for macroscopic realism beyond the Leggett-Garg inequalities," Phys. Rev. A 87, 052115 (2013).

32. A. R. Usha Devi, H. S. Karthik, Sudha, and A. K. Rajagopal, "Macrorealism from entropic Leggett-Garg inequalities," Phys. Rev. A 87, 052103 (2013).

33. C. Emary, F. Nori, and N. Lambert, "Leggett-Garg inequalities," Rep. Prog. Phys. 77, 016001 (2014).

34. A. Palacios-Laloy, F. Mallet, F. Nguyen, P. Bertet, D. Vion, D. Esteve, and A. N. Korotkov, "Experimental violation of a Bell's inequality in time with weak measurement," Nat. Phys. 6, 442-447 (2010).

35. V. Athalye, S. S. Roy, and T. S. Mahesh, "Investigation of the LeggettGarg inequality for precessing nuclear spins," Phys. Rev. Lett. 107, 130402 (2011).

36. G. Waldherr, P. Neumann, S. F. Huelga, F. Jelezko, and J. Wrachtrup, "Violation of a temporal Bell inequality for single spins in a diamond defect center," Phys. Rev. Lett. 107, 090401 (2011).

37. A. M. Souza, I. S. Oliveira, and R. S. Sarthour, "A scattering quantum circuit for measuring Bell's time inequality: a nuclear magnetic resonance demonstration using maximally mixed states," New J. Phys. 13, 053023 (2011).

38. G. C. Knee, S. Simmons, E. M. Gauger, J. J. L. Morton, H. Riemann, N. V. Abrosimov, P. Becker, H.-J. Pohl, K. M. Itoh, M. L. W. Thewalt, G. A. D. Briggs, and S. C. Benjaminl, "Violation of a Leggett-Garg inequality with ideal non-invasive measurements," Nat. Commun. 3, 606 (2012).

39. H. Katiyar, A. Shukla, K. R. K. Rao, and T. S. Mahesh, "Violation of entropic Leggett-Garg inequality in nuclear spins," Phys. Rev. A 87, 052102 (2013)

40. J. P. Paz and G. Mahler, "Proposed test for temporal Bell inequalities," Phys. Rev. Lett. 71, 3235-3239 (1993).

41. The Leggett-Garg inequality or the temporal Bell inequality involves linear combinations of correlations between temporally separated observables and is violated by almost every dynamically evolving quantum system (with an appropriate choice of observable measured at different intervals of time).

42. H. S. Karthik, A. R. Usha Devi, J. P. Tej, and A. K. Rajagopal, "Entropic uncertainty assisted by temporal memory," arXiv:1310.5079 (2013).

43. Y. N. Chen, C. M. Li, N. Lambert, S. L. Chen, Y. Ota, G. Y. Chen, and F. Nori, "Temporal steering inequality," Phys. Rev. A 89, 032112 (2014).

44. Temporal steering inequalities could be formulated by considering measurements of observables at different time instants in a single quantum system, as a natural temporal counterpart of EPR nonlocal steering inequalities. Recently, Chen et al. [43] investigated the violation of the temporal analog of a quadratic EPR steering inequality [see D. H. Smith, G. Gillett, M. P. Almeida, C. Branciard, A. Fedrizzi, T. J. Weinhold, A. Lita, B. Calkins, T. Gerrits, H. M. Wiseman, 
S. W. Nam, and A. G. White, "Conclusive quantum steering with superconducting transition-edge sensors," Nat. Commun. 3, 625 (2012)] given by $S_{N}=\sum_{i=1}^{3}\left\{\sum_{a= \pm 1} p(a)\left\langle B_{i}\right\rangle_{A_{i}=a}^{2}\right\} \leq 1$, where $\left\langle B_{i}\right\rangle_{A_{i}=a}=$ $\sum_{b} p(b \mid a) b ; A_{i}, B_{i}$ are qubit observables with binary outcomes $a, b= \pm 1$ in $N=2,3$ mutually unbiased bases, and $S_{N}$ denotes the steering parameter.

45. One could also extend the temporal steering protocol in a single quantum system, by considering measurements of any arbitrary noncommuting observables $X_{k}$ (which need not be associated with dynamical observable $X$ at time $t_{k}$ ). We have emphasized the temporal scenario here to highlight a natural counterpart of the EPR nonlocal steering (in spatially separated systems) for time-separated observables in a single quantum system.

46. Note that $\rho_{x_{k} \mid k}=\frac{1}{d} E_{k}\left(x_{k}\right)>0$ and the set of operators $\left\{d \rho_{x_{k} \mid k}\right\}$ obey $\sum_{k} d \rho_{x_{k} \mid k}=\sum_{k} E_{k}\left(x_{k}\right)=1$.

47. Necessary and sufficient conditions for the joint measurability of a generic set of $N$ dichotomic qubit POVMs $\left\{E_{k}\left(x_{k}\right), x_{k}=\right.$ $\pm 1 ; k=1,2, \ldots, N\}$ have been derived in Refs. [11,14] by identifying the range of the unsharpness parameter $\eta$ for which the POVMs are compatible/incompatible.

48. Invasiveness of measurements of an observable at different instants in time can be identified to be the temporal analog of nonlocality of spatially separated systems. 